\title{
EFFECTS OF MATRINE ON JAK-STAT SIGNALING TRANSDUCTION PATHWAYS IN BLEOMYCIN-INDUCED PULMONARY FIBROSIS
}

\author{
Xiuqin Ma ${ }^{1}$, Ruhua Chen ${ }^{1}$, Xiufang Liu ${ }^{1}, \mathrm{Jin} \mathrm{Xie}^{1},{\mathrm{Keyun} \mathrm{Si}^{1} \text { and Lirong Duan }}^{2}$ \\ ${ }^{1}$ Department of Respiratory Disease, Yixing People Hospital, Yixing 214200, China; \\ ${ }^{2}$ Colleges of Medicine Jiangsu University, Zhenjiang 212001, China \\ *E-mail: ruhuachencn@126.com
}

\begin{abstract}
The current study aims to investigate the effects of matrine on the JAK-STAT signaling transduction pathways in bleomycin (BLM)-induced pulmonary fibrosis (PF) and to explore its action mechanism. A total of 72 male C57BL/6 mice were randomized into the control, model, and treatment groups. PF models were established by instilling BLM intratracheally. The treatment group was given daily matrine through gastric lavage. Six mice were sacrificed in each group at 3, 7, 14, and 28 days. The lung tissues were observed using hematoxylin-eosin staining. The expression of JAK, STAT1, and STAT3 was observed using immunohistochemistry and then determined using real-time polymerase chain reaction. Alveolitis and PF significantly improved in the treatment group compared with the model group $(P<0.05)$. The expression of JAK, STAT1, and STAT3 in the model group increased at day 7, peaked at day 14 and then decreased, but the expression was still higher than that in the control group at day $28 \quad(P<0.05)$. The three indices in the treatment group were significantly lower than those in the model group at any detection time point $(P<0.05)$. PF causes high expression of JAK, STAT1, and STAT3. Matrine exerts an anti-PF effect by inhibiting the JAK-STAT signaling transduction pathways.
\end{abstract}

Key words: Pulmonary fibrosis, bleomycin, matrine, signaling transduction pathway.

\section{Introduction}

Idiopathic pulmonary fibrosis (PF) is primarily manifested by interstitial cell hyperplasia and the excessive deposition of extracellular matrixes dominated by collagen proteins (Gross and Hunningghake, 2001). The incidence of IPF has shown an increasing trend in recent years, but effective therapies aiming at increasing the survival rate of IPF patients and their clinical symptoms have not yet been confirmed. The fast development of the techniques in cytology and molecular biology has shed new light on the pathogenesis of PF (Hwang et al., 2005; Jack et al., 1990; Kaminski and Rosas, 2006). Signal transducers and activators of transcription (STATs) are a group of transcription factors which are activated by polypeptide ligands such as cytokines, as well as important substrates of receptor associated protein tyrosine kinases Janus Kinases/Just another Kinases (JAKs). Different JAK-STAT pathways are activated by different cytokines (Ratthe et al., 2007). Among these pathways, the activation of JAK-STAT3 is closely correlated with the occurrence of fibrotic diseases, thus, inhibitors of this pathway are promising to become the target of new drugs developed for these diseases (Ahn et al., 2009; Tu et al., 2005). Lightyellow sophora root is the dried root of Sophora flavescens ait (leguminosae). It is bitter in taste and cold in nature. Matrine is the major active component of the alkaloids of Sophora flavescens ait, and it has multiple pharmacologic actions and effects (Cao et al., 2010; Jiang et al., 2007; Liu et al., 2007; Yang et al., 2007). Matrine has a definite anti-liver fibrosis action and thus has been extensively applied in the treatments of chronic hepatitis, dermatofibrosis, and liver fibrosis (Gao and Law, 2009; Li et al., 2007). In the process of the initiation of PF, the mechanism of free radical injury plays a crucial role. 
Matrine can clear the free radicals with cytotoxic effect and inhibit the division and proliferation of firbroblasts thereby to exert anti-tissue damage and PF-preventing effects. However, whether matrine exerts these effects by influencing JAK-STAT signaling transduction pathways remains uncertain, and no report on this issue exists in literature.

In the current study, to investigate the specific effects of matrine on the JAK-STAT1 and JAK-STAT3 signaling transduction pathways, mouse models with PF were established with bleomycin (BLM). This study is intended to find the target of specific actions on the JAK-STAT signaling transduction pathways and to provide an experimental basis for the treatment of PF.

\section{Materials and Methods}

Animals

A total of 72 healthy male C57BL/6 mice, aging 8 weeks and weighing $20 \mathrm{~g}$ to $22 \mathrm{~g}$, were supplied by Shanghai Slac Laboratory Animal Co., Ltd., China. This study was conducted in strict accordance with the recommendations in the Guide for the Care and Use of Laboratory Animals of the National Institutes of Health, and the animal use protocol was reviewed and approved by the Institutional Animal Care and Use Committee (IACUC) of College of Medicine Jiangsu University.

\section{Grouping and medications}

The animals were randomized into the control, PF model, and treatment groups. The mouse was immobilized in a supine position after an abdominal anesthesia with $1.5 \%$ pentobarbital sodium. The neck was disinfected, and successive separation was performed to expose the trachea. An injector was inserted into the trachea in the direction of the heart through the tracheal cartilage ring gap for BLM infusion $(5 \mathrm{mg} / \mathrm{kg})$. The control group was given the same volume of physiological saline. After the infusion, the animal was straightened and rotated left and right immediately for an even distribution of the liquid in the lungs. At $24 \mathrm{~h}$ after model establishment, the control and model groups were subjected to gastric lavage with physiological saline, whereas the treatment group with matrine $(25 \mathrm{mg} / \mathrm{kg})$ (Ratthe et al., 2007) once daily. Six mice were killed in each group at $3,7,14$, and 28 days.

\section{Cell culture and inhibition action}

Human lung fibroblasts (LFs) MRC-5 were cultured with DMEM containing $10 \% \mathrm{FBS}$ in $5 \% \mathrm{CO}_{2}$ at $37{ }^{\circ} \mathrm{C}$. When the bottom of the culture bottle was covered by LF-like cells, $0.25 \%$ trypsin was applied for generations. The fourth generation was used. The cells were prepared into $1 \times 10^{4} / \mathrm{ml}$ monoplast suspension and then inoculated onto a 96-well plate for 24-h culture. They were divided into the control, model, and matrine $(0.8 \mathrm{mmol} / \mathrm{l})$ (Jiang and Xie, 2010) groups and then medicated separately for $1,3,5$, and 7 days. MTT solution at $20 \mu 1$, prepared with $5 \mathrm{mg} / \mathrm{ml}$ PBS of pH 7.4, was added into each well for $4 \mathrm{~h}$ of culture. The culture supernatant was discarded carefully (supernatants containing suspension cells had to be removed after centrifugation). $150 \mu 1$ of DMSO was added into each well. The solution was oscillated for 10 min to solve the crystals completely. The light absorption value of each well at a wavelength of $490 \mathrm{~nm}$ was read using an enzyme immunoassay instrument.

\section{Hematoxylin-eosin (HE) staining and immunohistochemistry}

After sacrifice, part of the lung tissue was fixed in $4 \%$ neutral formalin. It was routinely dehydrated, embedded and then sectioned for HE staining. Immunohistochemistry was performed for protein expression observation using the Strept avidin-biotin complex method. Part of the lung tissue was taken after sacrifice. It was fixed in $10 \%$ formalin overnight, 
http://dx.doi.org/10.4314/ajtcam.v10i3.10

routinely dehydrated, cleared, embedded in paraffin, serially sectioned $(5 \mu \mathrm{m})$, dewaxed and afterwards dried. The immuohistostaining of JAK, STAT1, and STAT3 was performed according to the instructions indicated in the kit (Wuhan Boster Bio-engineering Limited Company, China). Cells with brown- or buffy-stained cytoplasm or nucleus were recorded positive.

\section{Reverse transcription polymerase chain reaction (RT-PCR)}

Total RNA extraction and cDNA synthesis were performed according to kit instructions. Semi-quantitative PCR primers were designed according to JAK, STAT1, and STAT3 cDNA sequence in NCBI. The internal control GAPDH primer was 5'-AATCCCATCACCATCTTCCA-3 ' and 5'-CCTGCTTCACCACCTTCTTG-3'. For the PCR reaction, $1 \mu 1$ of cDNA, 2 $\mu \mathrm{l}$ of primer, and $10 \mu \mathrm{l}$ of $2 \times$ PCR Master Mix were added to $20 \mu \mathrm{l}$ of $\mathrm{ddH}_{2} \mathrm{O}$. The reaction conditions were as follows: $95{ }^{\circ} \mathrm{C}$ denaturation for $1 \mathrm{~min}, 95^{\circ} \mathrm{C}$ denaturation for $30 \mathrm{~s}, 60^{\circ} \mathrm{C}$ annealing for $30 \mathrm{~s}, 68^{\circ} \mathrm{C}$ extension for $90 \mathrm{~s}, 25$ cycles, and $68^{\circ} \mathrm{C}$ fully extended to $10 \mathrm{~min}$. The PCR products were detected using $1.5 \%$ agarose gel electrophoresis, and scanned by a gel imaging analysis system camera. The band density was analyzed by the ScnImage software.

\section{Statistical analysis}

Data were presented as means \pm standard error of means $(\mathrm{x} \pm \mathrm{s})$. They were analyzed using the SPSS12.0 software. Tukey's tes was performed to compare the mean values between groups.

\section{Results}

Inhibitory effect of matrine on LFs

Matrine had a significant inhibitory effect on LFs, compared with the model group $(P<0.05$; Table 1$)$.

\section{Pathological changes in the lung tissues}

In the control group, the lung tissues had clear structures without noticeable pathological changes. In the model group, noticeable alveolitis occurred in the tissues, with exudative changes in the pulmonary alveoli at day 3; at day 7 , exudation turned more serious and inflammatory cell infiltration appeared; from 14 day to 28 day, although alveolitis and exudation eased gradually, fibrosis aggravated gradually with noticeably increased alveolar septa, most alveolar structures were destroyed and pulmonary alveoli varied in volume. In the treatment group, the change from alveolitis to fibrosis also took place, but with a less degree, compared with that in the model group; inflammation and fibrosis were also less serious compared with those in the model group. The results are shown in Figure 1.

\section{The expression of JAK, STAT1, and STAT3}

In the control group, STAT1 and STAT3 were only lowly expressed in part of inflammatory cells. Compared with the control group, the model group exhibited noticeably deepened JAK and STAT1 staining: significantly increased expression in inflammatory cells and widely deeply-stained epithelial cells; STAT3 expression also greatly increased, which was principally localized in alveolar macrophages and partially localized in endothelial cells and neutrophils. In the treatment group, however, the expression of JAK, STAT1, and STAT3 significantly decreased compared with that in the model group. 


\section{RT-PCR}

The RT-PCR demonstrated that the mRNA expression of JAK, STAT1, and STAT3 in the lung tissues of the model

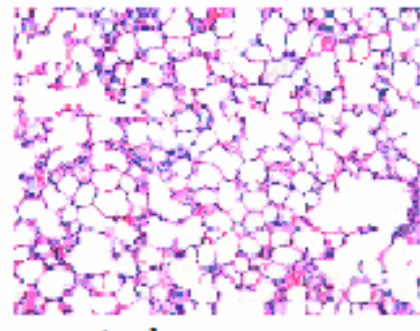
control group

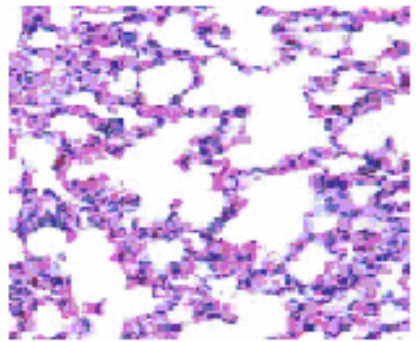

model group of 14 days

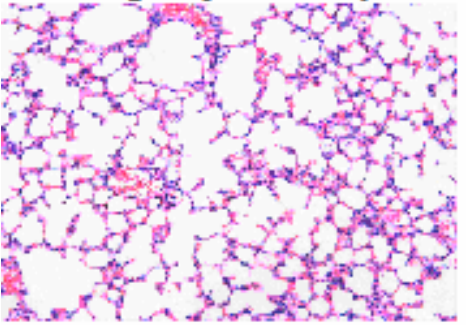

trentment group of 7 days

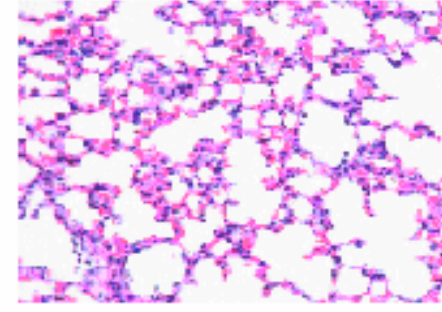

model group of 3 days

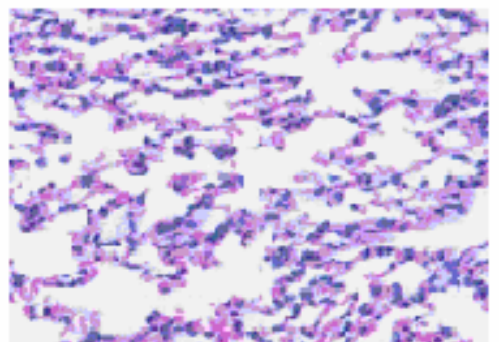

model group of 28 days

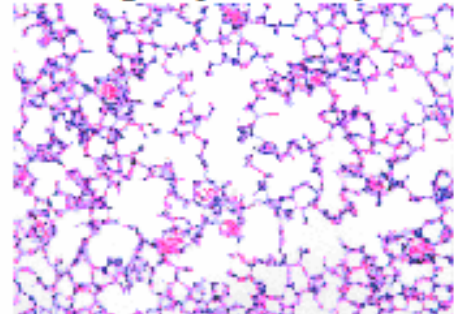

trentment group of 14 days

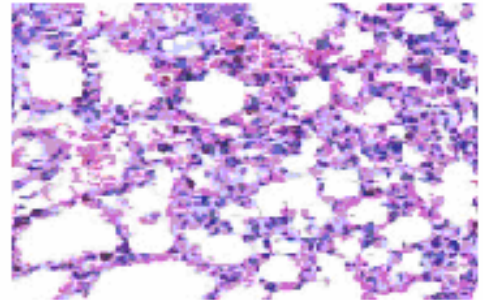

model group of 7 days

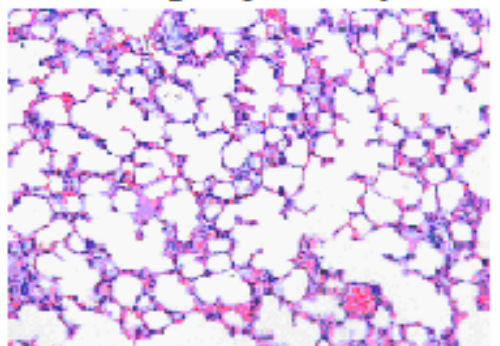

trentment group of 3 days
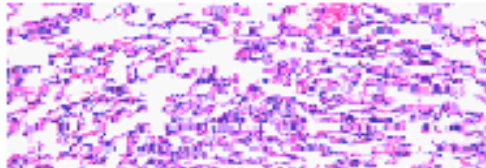

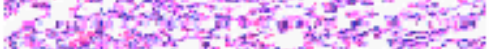
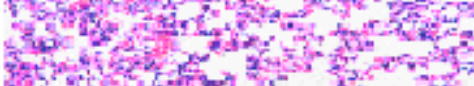

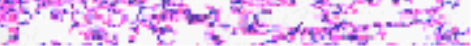

trentment group of 28 days

Figure 1: Pathological changes in the lung tissues (HE staining, $\times 400)$.

Table 1. Inhibitory effect of matrine on PF cells $(x \pm s, n=6)$

\begin{tabular}{lcccc}
\hline Groups & \multicolumn{4}{c}{ Time (d) } \\
& 1 & 3 & 5 & 7 \\
\hline Control & $0.227 \pm 0.022$ & $0.275 \pm 0.023$ & $0.237 \pm 0.030$ & $0.223 \pm 0.022$ \\
Model & $0.518 \pm 0.025^{*}$ & $0.747 \pm 0.027^{*}$ & $0.812 \pm 0.036^{*}$ & $0.892 \pm 0.025^{*}$ \\
Matrine & $0.502 \pm 0.027$ & $0.472 \pm 0.023^{* *}$ & $0.415 \pm 0.038^{* *}$ & $0.346 \pm 0.022^{* *}$ \\
\hline
\end{tabular}

${ }^{*} P<0.05$ compared with the control group, and $* * P<0.05$ compared with the model

group. 
Table 2 JAK mRNA expression of the control, model, and treatment groups $(x \pm \mathrm{s}, \mathrm{n}$ $=6)$

\begin{tabular}{ccccc}
\hline Groups & \multicolumn{4}{c}{ JAK mRNA expression } \\
& $7 \mathrm{~d}$ & $14 \mathrm{~d}$ & $21 \mathrm{~d}$ & $28 \mathrm{~d}$ \\
\hline Control & $0.214 \pm 0.026$ & $0.245 \pm 0.031$ & $0.235 \pm 0.023$ & $0.235 \pm 0.029$ \\
Model & $0.285 \pm 0.034$ & $0.982 \pm 0.125^{*}$ & $0.758 \pm 0.075^{*}$ & $0.613 \pm 0.075^{*}$ \\
Matrine & $0.178 \pm 0.021$ & $0.664 \pm 0.084^{* *}$ & $0.571 \pm 0.057^{* *}$ & $0.399 \pm 0.049^{* *}$ \\
\hline
\end{tabular}

$* P<0.05$ compared with the control group, and $* * P<0.05$ compared with the model group.

Table 3 STAT1 mRNA expression of the control, model, and treatment groups $\left({ }_{x}^{-} \pm \mathrm{s}\right.$, $\mathrm{n}=6$ )

\begin{tabular}{ccccc}
\hline Groups & \multicolumn{4}{c}{ STAT1 mRNA expression } \\
& $7 \mathrm{~d}$ & $14 \mathrm{~d}$ & $21 \mathrm{~d}$ & $28 \mathrm{~d}$ \\
\hline Control & $0.147 \pm 0.018$ & $0.168 \pm 0.015$ & $0.161 \pm 0.014$ & $0.161 \pm 0.019$ \\
Model & $0.195 \pm 0.023$ & $0.674 \pm 0.086^{*}$ & $0.521 \pm 0.022^{*}$ & $0.421 \pm 0.052^{*}$ \\
Matrine & $0.122 \pm 0.014$ & $0.456 \pm 0.058^{* *}$ & $0.392 \pm 0.023^{* *}$ & $0.274 \pm 0.033^{* *}$ \\
\hline
\end{tabular}

${ }^{*} P<0.05$ compared with the control group, and $* * P<0.05$ compared with the model group.

Table 4 STAT3 mRNA expression of the control, model, and treatment groups $(\bar{x} \pm \mathrm{s}$, $\mathrm{n}=6)$

\begin{tabular}{ccccc}
\hline Groups & \multicolumn{4}{c}{ STAT3 mRNA expression } \\
& $7 \mathrm{~d}$ & $14 \mathrm{~d}$ & $21 \mathrm{~d}$ & $28 \mathrm{~d}$ \\
\hline Control & $0.524 \pm 0.064$ & $0.599 \pm 0.076$ & $0.574 \pm 0.057$ & $0.574 \pm 0.071$ \\
Model & $0.696 \pm 0.085$ & $2.401 \pm 0.307^{*}$ & $1.854 \pm 0.185^{*}$ & $1.498 \pm 0.185^{*}$ \\
Matrine & $0.436 \pm 0.053$ & $1.624 \pm 0.207^{* *}$ & $1.397 \pm 0.139^{* *}$ & $0.976 \pm 0.121^{* *}$ \\
\hline
\end{tabular}

${ }^{*} P<0.05$ compared with the control group, and $* * P<0.05$ compared with the model group. 
group was significantly higher than that in the control group, whereas their mRNA expression in the treatment group was noticeably lower than that in the model group $(P<0.05)$. The results are shown in Tables $2-4$,.

\section{Discussion}

PF is associated with JAK-STAT signaling transduction pathways (Eitzman et al., 1996; Fan et al., 2003; Kim et al., 2002; Wang et al., 2009). These pathways are important cytokine signal transduction channels, which are involved in the gene regulation of cytokines in biological functions such as cytopoiesis, differentiation, embryo development, and organic immunity. JAKs are subject to a non-receptor type of tyrosine kinases and include JAK1, JAK2, JAK3, and Tyk2 as members. The members of JAKs and STATs activated by different cytokines display differences somewhat. Cytokines bind with their receptors and induce them to be dimerized to form combining sites for JAKs in cytoplasm; these sites activate JAKs, and then these activated JAKs activate STATs; the activated STATs undergo dimerization and nuclear localization and afterwards bind with specific DNA sequences to direct gene transcription (Meraz et al., 1996; Selman et al., 2004; Walters et al., 2005). These pathways conduct the intracellular signals of cytokines or even non-immunotransmitters, which involve varying pathophysiological processes such as immunity, cell proliferation and differentiation, apoptosis, inflammation, tumors, and so on. JAK-STAT signaling transduction pathways play an important role in the development of fibrosis.

The present study demonstrates that matrine has an obvious inhibitory effect on PF cells. This finding is consistent with what had been expected before the experiment. This study also showed that although the treatment group displayed a change from alveolitis to PF as well, its inflammation and PF lesion greatly improved compared with those in the model group. Furthermore, the immunohistochemistry and RT-PCR showed that JAK, STAT1, and STAT3 were abnormally expressed in the model group, which reached the peaks at $14 \mathrm{~d}$ and were significantly higher than those in the control group even at $28 \mathrm{~d}$. These findings suggest that the JAK-STAT1 and -STAT3 pathways play important roles in the development of early alveolitis and PF. Therefore, it is reasonable to presume that these pathways, through the abnormal expression of STAT1 and STAT3, induce the intra-pulmonary accumulation of inflammatory cells to activate the proliferation of smooth muscle cells and fibroblasts and then to increase collagen synthesis, ultimately leading to the occurrence of PF. In addition, this study shows that matrine can relieve the severity of alveolitis and PF and inhibit the growth of pulmonary fibrocytes by downregulating the abnormal expression of JAK, STAT1, and STAT3 to exert its anti-fibrosis function. This finding provides a new method for the treatment of PF. Furthermore, by observing the changes in various signaling molecules of the JAK-STAT pathways during the progression of PF, this study also provides a theoretical foundation for study on the roles of JAK-STAT pathways in the development of PF, as well as a new breakthrough point for further study on the molecular mechanism of PF. However, this study has a limitation: It merely based the influence of matrine on the JAK-STAT pathways in PF mice on the study of the expression of proteins and nucleic acid molecules using immunohistochemistry and RT-PCR. To further clarify the anti-PF mechanism of matrine, a planned study will use primarily cultured cells to determine the expression of various molecules in the JAK-STAT pathways at different stages of PF induced with BLM. That study will be valuable not only in proposing a new idea for exploration into the pathogenesis of PF but in offering a new target for PF treatment.

\section{References}

1. Ahn HJ, Kim JY, Ryu KJ, Nam HW (2009). STAT6 activation by Toxoplasmagondii infection induces the expression of Th2 C-C chemokine ligands and B clade serine protease inhibitors in macrophage. Parasitol. Res. 105: $1445-1453$.

2. Cao YG, Jing S, Li L, Gao JQ, Shen ZY, Liu Y, Xing Y, Wu ML, Wang Y, Xu CQ, Sun HL (2010). Antiarrhythmic effects and ionimechanisms of oxymatrine from Sophora flavescens. Phytother. Res. 24: 1844-1849.

3. Eitzman DT, McCoy RD, Zheng X, Fay WP, Shen T, Ginsburg D, Simon RH (1996). Bleomycin-induced 
pulmonary fibrosis in transgenic mice that either lack or overexpress the murine plasminogen activator inhibitor-1 gene. J. Clin. Invest. 97: 232-237.

4. Fan XM, Wang ZL, Li ZH (2003). STAT1 activation and STAT1-dependent immune response gene ICAM-1 expression in alveolar maerophages of rats sufered from interstitial pulmonary fibrosis. Chin. J. Cell. Mol. Immunol. 19: 3-6.

5. Gao G, Law FC (2009). physiologically based pharmacokinetics of matrine in the rat after oral administration of pure chemical and ACAPHA. Drug Metab. Dispos. 37: 884-891.

6. Gross TJ, Hunningghake GW (2001). Idiopathic pulmonary fibrosis. N. Engl. J. Med. 345: 517-525.

7. Hwang D, Smith JJ, Leslie DM, Weston AD, Rust AG, Ramsey S, de Atauri P, Siegel AF, Bolouri H, Aitchison JD, Hood L (2005). A data integration methodology for systems biology: experimental verificaion. Proc. Natl. Acad. Sci. U S A. 102: 17302-17307.

8. Jack A, Bruce F, Jeffrey A, Rosenbloom J (1990). Cytokine network in the regulation of inflammation and fibrosis in the lung. Chest 97: 1439-1445.

9. Jiang H, Hou C, Zhang S, Xie H, Zhou W, Jin Q, Cheng X, Qian R, Zhang X (2007). Matfine upregulates the cell cycle protein E2F-1 and triggers apoptosis via the mitochondrial pathway in K562 cells. Eur. J. Pharnlacol. 559: 98-108.

10. Jiang YY, Xie M (2010). Matrine Inhibits Cell Proliferation and Expression of CTGF and HIF-1a of Human Lung Fibroblast in Normoxia and Hypoxia. Chin. J. Respir. Crit. Care. Med. 9: 44-47.

11. Kaminski N, Rosas O (2006). Gene expression profiling as window into idiopathic pulmonary fibrosis pathogenesis: can we identify the right target genes. Proc. Am. Thorac. Soc. 3: 339-344.

12. Kim OS, Park EJ, Joe EH, Jou I (2002). JAK-STAT signaling mediates gangliosides induced inflammatory responses in brain microglial ceils. J. Biol. Chem. 277: 40594-40601.

13. Li Y, Wang B, Zhou C, Bi Y (2007). Matrine induces apoptosis in angiotensin II-stimulated hyperplasia of cardiac fibroblasts: effects on Bcl-2/Bax expressionand caspase -3 activation. Basic Clin. Pharmacol. Toxicol. 101: 1-8.

14. Liu JY, Hu JH, Zhu QG, Li FQ, Wang J, Sun HJ (2007). Efect of matrine on the expression of substance receptor and inflammatory cytokines production in human skin keratinocytes and fibroblasts. Int. Immunopharmacol. 7: 816-823.

15. Meraz MA, White JM, Sheehan KC, Bach EA, Rodig SJ, Dighe AS, Kaplan DH, Riley JK, Greenlund AC, Campbell D, Carver-Moore K, DuBois RN, Clark R, Aguet M, Schreiber RD (1996). Targeted disruption of the Statl gene in mice reveals unexpected physiologic specificity in the JAK-STAT signaling pathway. Cell 84: 431-442.

16. Ratthe C, Pelletier M, Chiasson S, Girard D (2007). Molecular mechanisms involved in interleukin-4 induced human neutrophils: expression and regulation of suppressor of cytokine signaling. J. Leukoc. Biol. 81: 1287-1296.

17. Selman M, Thannickal VJ, Pardo A, Zisman DA, Martinez FJ, Lynch JP 3rd (2004). Idiopathic pulmonary fibrosis: pathogenesis and therapeutic approaches. Drugs 64: 405-430.

18. Tu Y, Salim S, Bourgeois J, Di Leo V, Irvine EJ, Marshall JK, Perdue MH (2005). CD23-mediated IgE transportacross human intestinal epithelium: inhibition by blocking sites oftranslation or binding. Gastroenterology 129: 928-940.

19. Walters DM, Antao-Menezes A, Ingram JL, Rice AB, Nyska A, Tani Y, Kleeberger SR, Bonner JC (2005). Susceptibility of signal transducer and activator of transcription-1-deficient mice to pulmonary fibrogenesis. Am. J. Pathol. 167: 1221-1229.

20. Wang WJ, Liao B, Zeng M, Zhu C, Fan XM (2009). The effects of aerosolized stat1 antisense oligodeoxynucleotides on rat pulmonary fibrosis. Cell. Mol. Immunol. 6: 51-59.

21. Yang JJ, Gao SM, Chen H (2007). Apoptosis of multiple myeloma RPMI8226 cell line induced by matrine. Int. 
Ma et al., Afr J Tradit Complement Altern Med. (2013) 10(3):442-448

http://dx.doi.org/10.4314/ajtcam.v10i3.10

Immunopharmacol. 15: 515-518. 